\title{
Editorial
}

\section{Another genotoxic agent released by mitochondrial meltdown}

\author{
RA Knight ${ }^{\star, 1}$ and PX Petit ${ }^{\star, 2}$ \\ ${ }^{1}$ Department of Cystic Fibrosis, National Heart and Lung Institute, Imperial \\ College, Manresa Road, London SW3 6LR, UK \\ 2 Cochin Institut of Molecular Genetics, INSERM/CNRS-Department of Genetics, \\ Development and Molecular Pathology - CHU Cochin Port-Royal, 24, rue du \\ Faubourg Saint-Jacques, F-75014, Paris \\ * Corresponding authors: e-mail: r.a.knight@ic.ac.uk/ \\ pxpetit@zeus.cochin.inserm.fr
}

It has been suggested that active death and survival signals originated with the acquisition by bacteria of a toxin/antitoxin package, the toxin killing competing bacteria and the antitoxin protecting the host bacterium from killing itself at the same time. ${ }^{1}$ Similarly, eukaryotic cells are thought to have acquired mitochondria through the endosymbiotic incorporation of bacteria - with their toxin/antitoxin module - with intracellular specialisation of mitochondria into the cellular power house. Does mitochondrial damage result in dysregulation of its ancestral toxin/antitoxin component with consequent death of the cell? However, recent studies of unicellular eukaryotes (protist), some of them little known, have provided insights that challenge the traditional serial endosymbiosis based view of how the eukaryotic cell and its mitochondria came to be. These data indicate that the mitochondrion arose in a common ancestor of all extant eukaryotes and raise the possibility that this organelle originated at essentially the same time as the nuclear component of the eukaryotic cell rather than in a separate, subsequent event. ${ }^{2}$ This modern view, that the cell death component may have evolved from the simple establishment and success (stabilization mechanisms?) of the original symbiotic association ( $\alpha$-proteobacteria and an Archae $)^{2}$ may support a new and original view point on the establishment of cell death mechanisms.

To be simplistic, when a nuclear power station blows up - as happened, for example, at Six Mile Island and Chernobyl - we need to know two things to bring the problem under control. How do we seal off the damaged reactor, and what harmful material has already leaked. The same questions apply when the cellular power station the mitochondrion - goes out of control. With the nuclear power plant, we use both specific and non-specific shutdown procedures. We drop boron rods into the dysfunctional reactor and, panicking, force emergency units to spray sand and concrete containment. The same with the damaged mitochondrion; overexpress Bcl-2 and rush in pharmacological concentrations of z-VADfmk. And, in the same way as physicists must analyze the nature of the radioactive cloud released when a reactor blows for its DNA-damaging and other harmful contents, so biologists have to identify the DNA-damaging and other harmful molecules released following a mitochondrial insult. The problem facing the biologist is, however, far more complex than that posed by a rogue reactor.

Three recent papers, two in Nature ${ }^{3,4}$ and one in this issue of $\mathrm{CDD},{ }^{5}$ now add a new protein, endoG, to the cytosolic molecular pollution caused by mitochondrial damage. EndoG, and its $C$ elegans homologue, cps-6, are proteins of relative molecular masses of about $30 \mathrm{kDa}$. They are encoded in the nuclear genome, contain a mitochondrial localisation signal and seem to be confined to the mitochondrial intermembrane space. The nematode protein shares $48 \%$ identity and $69 \%$ similarity with mammalian endoG, and also has significant similarity with a predicted Drosophila sequence. EndoG is therefore a member of an evolutionarily conserved pro-apoptotic family, this very conservation implying biological importance. Treatment of mitochondria with the cleaved active form of Bid in vitro, and administration of agonistic anti-Fas antibodies in vivo, cause translocation of endoG into the cytosol and into the nucleus, an effect blocked by over expression of $\mathrm{Bcl}-2$. In vitro, endoG produces internucleosomal fragmentation of nuclear DNA, and this endonuclease activity is expressed without any requirement for caspases. EndoG therefore joins a distinguished cast of mitochondrial characters such as AIF, ${ }^{6,7}$ cytochrome $c{ }^{8}$ and Smac/Diablo, ${ }^{9,10}$ which can destroy the cell if mitochondrial integrity is compromised. Perhaps we can now begin to see that these mitochondrial toxins form a hierarchy, with cytochrome c/Apaf-1/procaspase 9 causing cellular damage through caspase-mediated proteolysis, Smac/Diablo inhibiting the IAP antitoxins and AIF (antagonized by $\mathrm{Hsp} 7 \mathrm{O}^{11}$ ) and endoG being involved in the nuclear DNA disagregation, the ultimate destroyers of nuclear DNA. Moreover, if these mitochondrial toxins are echoes of an ancient toxin/antitoxin module, we can ask whether proteins like endoG have orthologues in bacteria. If survival of the eukaryotic cell depends, at least partly, on a positive social dialogue between resident nucleus and acquired mitochondrion (first hypothesis), and if any disturbance of successful symbiosis interrupts intracellular survival signalling and activates default death pathways, then endoG may be one knife in the nuclear back. The mitochondrion kills the cell that nurtures it by releasing endoG?

Well, perhaps not by itself. $\mathrm{AIF}^{7}$ another protein restricted to the intermembrane space in intact mitochondria and apparently conserved during evolution, ${ }^{12-14}$ is also released following mitochondrial injury, and causes high molecular weight DNA fragmentation and chromatin condensation in a caspase-independent manner. If, then, endoG is the knife that cuts DNA into 180 bp oligonucleosomal fragments, is AIF the axe that performs the preceding large scale cleavage? Although the data 
published from Xiaodong Wang and Peter Vandenabeele's labs suggest that, in these rather artificial systems, and at rather high concentration, endoG can act alone, physiologically do the two mitochondrial proteins work sequentially, and how do they interact at a molecular level? Do they also destroy mitochondrial DNA?

Several other non-mitochondrial DNAses have also been implicated in classic apoptotic nuclear DNA fragmentation, including DNAsel, DNAsell, DNAse $\gamma$ and DNAseX, although these do not meet all the criteria for an apoptotic DNAse. A more likely candidate is CAD (DFF40) ${ }^{15,16}$ which forms an inactive heterodimer with its inhibitor, ICAD. Caspase-3-mediated cleavage of ICAD releases the active $\mathrm{CAD}$, which translocates from the cytosol to the nucleus where it causes internucleosomal DNA fragmentation. Unlike AIF and endoG, therefore, CAD activation is caspase-dependent. ICAD knockout mice are deficient in CAD activity, probably because ICAD is required to correctly chaperone CAD, and these mice show reduced DNA fragmentation in response to a number of apoptotic stimuli. ${ }^{17}$ The knockout animals, however, develop normally, particularly in their immune system, in which the dysfunctional $90 \%$ of immature thymocytes are destined to die by apoptosis. Clearly, CAD cannot be the only physiological DNAse. Is this the role of AIF and endoG?

The activation of CAD appears to be a downstream event following death receptor ligation and activation of caspases-8 and -3 . Caspase-mediated cleavage and activation of Bid would then lead to mitochondrial release of AIF and endoG. Activation of the intrinsic death pathway would only release AIF and endoG, and DNA fragmentation produced by mitochondrial injury alone would appear to be independent of CAD. However, the available experimental data may argue against this simplification. While recombinant $\mathrm{CAD}$ is active in vitro in $\mathrm{nM}$ quantities, the mitochondrial endonuclease seems only to fragment DNA at much higher concentrations.

If the final definition of the death of a cellular society is the destruction of its DNA library, then in endoG we have a persuasive molecular mediator released when the mitochondrial power house explodes. But we still need to know whether endoG can cause death by itself - does it require AIF? Can it fragment DNA in the absence of caspasemediated cleavage of substrates such as PARP and lamin B1? - as, apparently, can CAD. The absence of hallmarks of apoptosis - including DNA fragmentation-in cells treated both with a mitochondrial damaging agent such as staurosporine and a broad spectrum caspase inhibitor would, however, argue that endoG (and AIF) do not act in isolation. May be we should now turn our attention from identifying the individual components leaking from the blown power station to studying their interactions.

1. Ameisen JC (2002) Cell Death Differ. 9: (in press)

2. Gray MW et al. (2001) Genome Biol. 2: 1081.1-1081.6

3. Li LY et al. (2001) Nature 412: $95-99$

4. Parrish J et al. (2001) Nature 412: 90-94

5. van Loo G et al. (2001) Cell Death Differ. 8: 1136-1142

6. Susin SA et al. (2001) J. Exp. Med. 184: 1331-1341

7. Susin SA et al. (1999) Nature 397: 441-445

8. Liu X et al. (1996) Cell 86: 147-157

9. Du C et al. (2000) Cell 102: 33-42

10. Verhaghen AM et al. (2000) Cell 102: $43-53$

11. Ravagnan L et al. (2001) Nature Cell Biol. 3(9): 839-843

12. Lorenzo HK et al. (1999) Cell Death Differ. 6: 516-524

13. Arnoult D et al. (2001) Mol Biol. Cell 12: $3016-3030$

14. Arnoult $\mathrm{D}$ et al. (2002) Cell Death Differ 9: (in press)

15. Liu X et al. (1997) Cell 89: 175-184

16. Enari M et al. (1998) Nature 391: 43-50

17. Zhang J et al. (1998) Proc. Natl. Acad. Sci. USA 95: 12480-12485 